\title{
Scale Alignment in Between-Item Multidimensional Rasch Models
}

LEAH FEUERSTAHLER is an Assistant Professor of Psychometrics and Quantitative Psychology at Fordham University, 441 East Fordham Rd., Bronx, NY 10458; 1feuerstahler@fordham.edu. Her primary research interests include item response theory and psychological measurement.

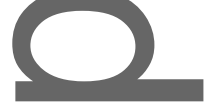

"MARK WILSON is a Professor in the Graduate School of Education at UC Berkeley, Berkeley, CA 947 20 ; MarkW@berkeley.edu. His primary research interests are in psychometrics, educational assessment, and philosophy of measurement.

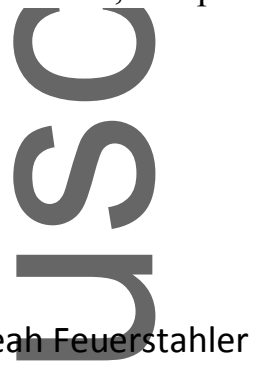

Job Title: Assistant Professor of Psychometrics and Quantitative Psychology

Affiliation: Fordham University

Address: Department of Psychology
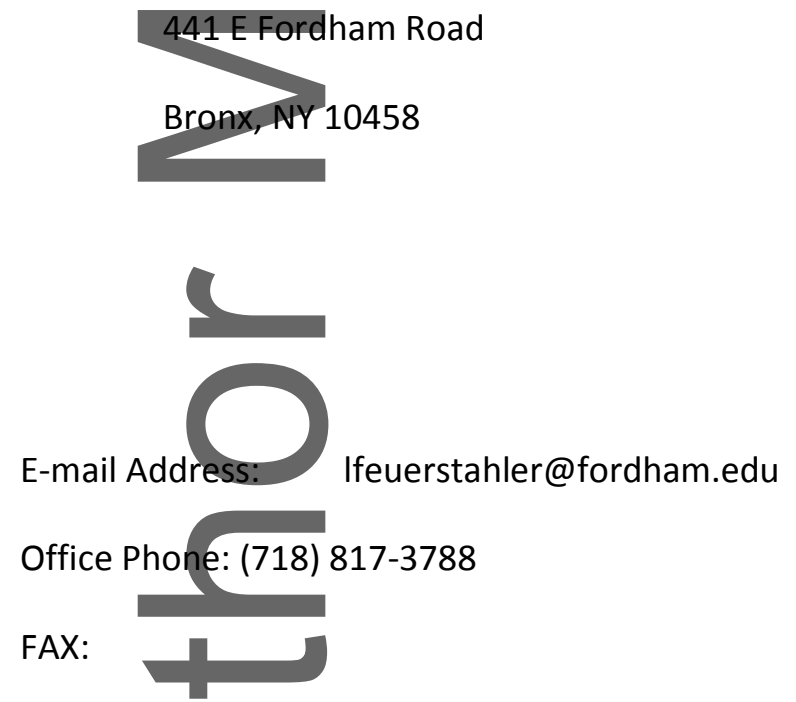

Areas of Specialization: item response theory, psychometrics, statistics

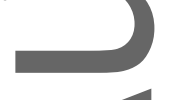

This is thenth r manuscript accepted for publication and has undergone full peer review but has not been through tho pyediting, typesetting, pagination and proofreading process, which may lead to differences between this version and the Version of Record. Please cite this article as doi:

10.1111/jedm.12209.

This article is protected by copyright. All rights reserved. 


\begin{abstract}
Scores estimated from multidimensional item response theory (IRT) models are not necessarily comparable across dimensions. In this paper, the concept of aligned dimensions is formalized in the context of Rasch models, and two methods are described - delta dimensionah alignment (DDA) and logistic regression alignment (LRA) - to transform estimated item parameters so that dimensions are aligned. Both the DDA and LRA methods are applied to real and simulated data, and it is demonstrated that both methods are broadly effective for achieving aligned scales. The routine use of scale alignment methods is recommended prior to comparing scores across dimensions.

In many assessment contexts, researchers wish to measure multiple distinct constructs
\end{abstract} in the same population. Historically, this required modeling each measured dimension separately with either classical or unidimensional item response theory (IRT) methods. More recently, multidimensional item response models have been developed that allow multiple measurement dimensions to be modeled simultaneously (e.g., Adams, Wilson, \& Wang, 1997; McKinley \& Reckase, 1983; Whitely, 1980). Recent innovations in IRT software and computational speed have permitted the widespread application of multidimensional IRT to data gathered from moderate to large samples. Compared to separate (consecutive) estimation of each dimension, between-item multidimensional IRT models, under which each item belongs to exactly one dimension, allow dimensions to be more reliably estimated because of shared information across correlated dimensions. That is, information about the correlation among dimensions can be used to improve the precision of parameter estimates, even if some dimensions include only a small number of items. Multidimensional IRT has the additional advantage of directly estimating the disattenuated correlation among dimensions (Adams, et al., This article is protected by copyright. All rights reserved. 
1997).

When simultaneously measuring multiple dimensions, a common substantive question is how an individual or a group of individuals perform on one dimension relative to another dimension that is related but qualitatively distinct (c.f., Angoff's (1971) discussion of comparable scores). Such comparisons are appropriate when dimensions are meaningfully related, for example, in contexts for which it is sensible to report composite scores. As a concrete example, consider an achievement test that contains both science and mathematics items. Even though these tests measure different constructs, it is sensible to ask questions such as "which test is more difficult?" and "is a particular student more proficient in science than in mathematics?". Unfortunately, traditional procedures do not provide straightforward answers to these questions. The raw (number correct) scores on the science and mathematics tests are not necessarily on comparable metrics because tests can vary in difficulty, and the distribution of student abilities might vary across the two tests. The interpretational problem of comparing seores on the two dimensions persists when using IRT logit score estimates rather than raw scores. As will be detailed in a later section, fitting item response models requires specifying model identification constraints, which are almost always specified separately for each dimension in a multidimensional analysis. In other words, multidimensional identification constraints may not lead to dimensions that are directly comparable to each other.

The purpose of the current work is to propose a criterion for scaling multiple dimensions so that dimension scores can be compared. These procedures will be referred to as scale alignment methods. Under these methods, rather than modifying the model identification constraints for multidimensional model estimation, linear transformations (similar to those used to link separate calibrations) are applied to item parameters after estimation.

This article is protected by copyright. All rights reserved. 
Before reviewing the history of similar methods, the concept of scale alignment is illustrated in Figure 1. To create this figure, item response data were simulated under a twodimensional Rasch model for which each item belongs to only one dimension (between-items multidimensionality). These data were then fit to the data-generating model using the TAM (Robitzsch, Kiefer, \& Wu, 2017) package for R (R Core Team, 2018), and by constraining person means to equal zero on both dimensions. This figure displays estimated item response functions for two items belonging to two different dimensions with the same value for their sufficient statistics for the same sample of persons. The first two panels of Figure 1 show the item response functions for the selected items and the marginal latent trait distributions for each dimension. For this data set, these two items have the same proportion of correct responses $(p=.84)$, but different estimated item difficulties $\hat{\delta}_{1}=-1.95$ and $\hat{\delta}_{2}=-2.62$. Note that it is almost always the case that items with the same sufficient statistic will have different item difficulty estimates for different dimensions. In the final panel of Figure 1, the two items are ptaeed on aligned scales, where the " $\sim$ " symbol is used to denote alignment, such that the same proportion correct leads to the same estimated item difficulties on both dimensions. In this example, $\tilde{\hat{\delta}}_{1}=\tilde{\hat{\delta}}_{2}=-2.03$. In other words, for aligned scales, items for which examinees (as a group) perform equally well have identical item parameter estimates, regardless of dimension.

Scale alignment is conceptually related to battery scaling methods, according to the taxonomy of sealing methods proposed by Holland and Dorans (2006). These authors use the term battery scaling to refer to methods intended to put scales representing different constructs onto comparable metrics. However, the ideas behind battery scaling appear much earlier in the literature. Nearly a century ago, Kelley (1923) described methods to achieve 
comparable measures of the distinct constructs of intelligence and achievement. Later, the term comparable scores was used to describe scores on related constructs such as reading and mathematics (Angoff, 1971; Flanagan, 1951) that have been scaled to have the same score distribution in a given population. The most prominent example of battery scaling is the alignment of the Verbal and Mathematical subtests of the scholastic aptitude test (SAT-V and SAT-M; Dorans, 2002). Battery scaling of the SAT-V and SAT-M was done with reference to the full distribution of scores on each dimension. In this and other instances of battery scaling, scale scores were transformed so that, for a representative population, the distributions of transformed scores were approximately equal for all scales. These transformations used equipercentile equating methods which apply nonlinear transformations to scaled scores.

To apply battery scaling ideas in a Rasch context requires different methods for two main reasons. First, within a Rasch modeling framework, only linear metric transformations are appropriate. In contrast, the transformations required by equipercentile equating are typically nonlinear. Second, item parameters should be placed on the transformed scales, not only person parameters. Because Rasch model item and person parameters are on the same scale, scaling transformations should result in both types of parameters being comparable across dimensions. Notably, the classical item difficulty - that is, the proportion of examinees who answer an item correctly - already exists as an intuitive way to compare item locations on different dimensions. This statistic can be used to determine which items are more difficult than others, even if the items being compared belong to different dimensions. As will be seen in a later section, our proposed methods for IRT-based scale alignment rely on the comparability of estimated item parameters across dimensions. Thus, although scale alignment is similar to battery scaling in purpose and in the fact that both approaches are This article is protected by copyright. All rights reserved. 
sample-specific, the methods proposed in this paper are unlike those used for battery scaling.

We prefer to use the term sample-specific rather than norm-referenced when referring to scale alignment. Norm-referencing typically refers to methods that directly reference a distribution of examinees. This type of norm-referencing is prevalent in multidimensional latent variable modeling. For example, most applications of exploratory and confirmatory factor analysis standardize the latent variables. The consequence of this standardization process is that the means and variances of the sample scores are set equal on each dimension. As a result, score estimates are interpreted relative to a standardized distribution of scores on each dimension. The multidimensional Rasch model is free from some of the scaling assumptions of factor analysis. Whereas factor analysis models are usually identified by setting latent variable means to zero and variances to one, the latent variable variances can be freely estimated for Rasch models. However, some assumptions are required to identify the origin of each dimension. This can be done (a) by fixing the average person location to zero, (b) by fixing the average item difficulty to zero, or (c) by anchoring to pre-calibrated item or person parameters. Often the identification restrictions are chosen for convenience rather than for substantive psychological reasons. In addition, for between-item multidimensional Rasch models, these identification restrictions are effectively separate for each dimension. For this reason, there is no clear basis for which to directly compare scores across dimensions.

The problem of comparing scores on different multidimensional IRT dimensions was first identified by Yamada, Draney, Karelitz, Moore, and Wilson (2006; see also Schwartz, 2012; Schwartz \& Ayers, 2011). These authors proposed a scale alignment method originally called the delta teehnique (Yamada et al., 2006) and later referred to as delta-dimensional alignment (DDA; Schwartz, 2012; Schwartz \& Ayers, 2011). The DDA method builds on the idea of a reference dimension, a single dimension that is a composite of all dimensions This article is protected by copyright. All rights reserved. 
represented by the data (Ackerman, 1988, 1992; Wang, 1986). As will be detailed in a later section, under the DDA method, the distributions of item parameters when data are fit to a unidimensional model is used to transform multidimensional item parameters into alignment. The DDA method has since been used to align dimensions of teacher candidate proficiency (Castellano, Duckor, Wihardini, Tellez, \& Wilson, 2016), knowledge of structure of matter concepts in middle school science (Morell, Collier, Black, \& Wilson, 2017), and scientific content knowledge with scientific argumentation ability (Osborne, Henderson, MacPherson, Szu, Wild, \& Yao, 2016; Yao, Wilson, Henderson, \& Osborne, 2015). The need for aligning multiple dimensions was later independently identified by Twiss and McKenna (2015). These authors suggested aligning dimensions using a multidimensional Rasch model under which some items load on multiple dimensions (within-item multidimensionality). Twiss and McKenna argue that allowing some items to load on multiple dimensions automatically aligns dimensions so that direct comparisons are warranted. However, as the preliminary use of the DDA method shows, common items across dimensions are not necessary for alignment, and the remainder of this paper discusses scale alignment in the context of between-items multidimensional Rasch models.

The goal of this paper is to formalize a set of methods for aligning multidimensional IRT scales sueh that direct comparisons across dimensions are warranted. Specifically, a formal definition for aligned dimensions is proposed for use under multidimensional Rasch models. Based on this definition, a modification of the original DDA method is described, and a second alignment method called logistic regression alignment (LRA) is developed.

Admissible Transformations of the Between-Items Multidimensional Rasch Model The unidimensional Rasch model for dichotomous data is defined as follows. For This article is protected by copyright. All rights reserved. 
person $j$ and item $i$, the probability $P$ of a correct response equals

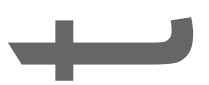

$$
\operatorname{logit}\left(P_{i j}\right)=\theta_{j}-\delta_{i}
$$

where $\theta$ is a person location parameter and $\delta$ is an item location parameter. Equivalently, the Rasch model can be written

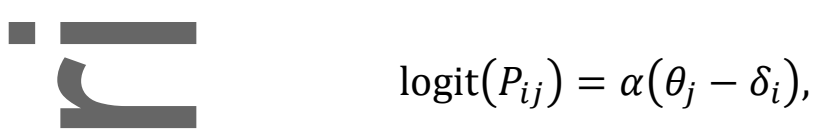

where $\alpha$ controls the steepness of the item characteristic curve and is usually set equal to 1 . It should be emphasized that the models expressed in Equations (1) and (2) are identical, even if $\alpha \neq 1$, so long as $\alpha$ does not vary across items. Specifying $\alpha \neq 1$ simply affects the unit size of the latent variable. As a result, the latent variance for the model specified in Equation (1) will differ from the latent variance for the model specified in Equation (2) if $\alpha \neq 1$.

In Equation (2), suppose that $\alpha$ is set to a constant for a set of items. It is also possible to set $\alpha$ to different constants for subsets of pre-specified items (e.g., Hoijtink, Rooks, \& Wilmink,1999). Recent research has suggested that, within a test, $\alpha$ may vary for sets of items due to empirical factors (Bolsinova, Maris, \& Hoijtink, 2016; Humphrey, 2011) or across groups of persons (Humphrey, 2010). For a between-items multidimensional Rasch model, it is also possible to let $\alpha$ vary across dimensions (Gochyyev, 2015). Specifically, if item $i$ belongs to dimension $d$,

$$
\operatorname{logit}\left(P_{i(d) j}\right)=\alpha_{d}\left(\theta_{d j}-\delta_{i(d)}\right)
$$

where $\alpha_{d}$ is the steepness parameter for dimension $d, \theta_{d j}$ is the ability of person $j$ on dimension $d$, and $\delta_{i(d)}$ is the location of item $i$ belonging to dimension $d$.

A general form for the between-items multidimensional Rasch model is shown in Figure 2, where $\sigma_{d}^{2}$ indicates the latent variable variance on dimension $d$. Notice in this figure that each dimension can have a different steepness parameter $\alpha_{d}$ and a different latent variable variance This article is protected by copyright. All rights reserved. 
$\sigma_{d}^{2}$. As with unidimensional models, multidimensional Rasch models are typically specified by setting all $\alpha$ values to 1 . Gochyyev (2015) suggested instead setting dimension variances, $\sigma_{d}^{2}$, equal to 1 and estimating the $\alpha_{d}$ parameters. In this solution, if latent variable means are all set equal to zero, this model effectively standardizes the latent variables. If instead scales are to be aligned based on properties of the items, then the general specification of the between-items multidimensional Rasch model shown in Figure 2 and given in Equation (3) is needed to align scales. In this paper it is assumed that data are generated and models are fit using the convention that $\alpha_{d}=1$ for all dimensions. Then, the general model given in Equation (3) is used to express the model after scale alignment.

To transform any set of item or dimension parameters $\alpha_{d}, \theta_{d j}$, and $\delta_{i(d)}$ to a transformed set of parameters $\tilde{\alpha}_{d}, \tilde{\theta}_{d j}$, and $\tilde{\delta}_{i(d)}$ (Davey, Oshima, \& Lee, 1996, 2000) requires two constants per dimension: a scaling constant $r_{d}$ and a shift constant $s_{d}$ such that

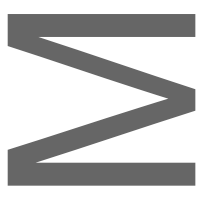

and

$$
\tilde{\delta}_{i(d)}=r_{d} \delta_{i(d)}+s_{d} \text {. }
$$

The transformations shown in Equations (4) - (6) may be applied separately for each dimension, and these transformations affect the distribution of $\tilde{\theta}_{d}$. If the original dimension $d$ has mean $\mu_{d}$ and variance $\sigma_{d}^{2}$, then the transformed dimension $d$ has mean $r_{d} \mu_{d}+s_{d}$ and variance $r_{d}^{2} \sigma_{d}^{2}$.

Before setting forth a definition of aligned scales, the purpose of scale alignment should be clarified in a broader context. It is well-known that the IRT latent trait metric does

This article is protected by copyright. All rights reserved. 


\section{SCALE ALIGNMENT}

not provide an absolute (ratio-level) metric. Therefore, the metric must be anchored using identification constraints that are usually chosen out of convention or convenience rather than substantive theory. Different (but linearly related) scales can be defined by choosing other identification constraints or by making appropriate transformations after using any constraints. There is no mathematical reason to choose one set of identification constraints over anothen Transforming parameters using the equations presented in this paper will have absolutely no effect on the fit of the data to the model. In this respect, any of these solutions are admissible. The notion of scale alignment merely suggests that some of these admissible solutions are better for interpretation and comparison than others. In this respect, scale alignment serves a similar role in between-items multidimensional IRT that factor rotation serves in exploratory factor analysis (EFA). In EFA, factor rotation does not affect modeldata fit. Instead, most factor rotation methods aim to satisfy the idea of simple structure (Thurstone, 1935), which should lead to a more interpretable factor pattern than the unrotated factor pattern. Similarly, score comparisons across dimensions are interpreted more appropriately for aligned scales than for unaligned scales.

\section{Scale Alignment}

When between-items multidimensional IRT models are identified by constraining item or person parameter means equal to zero, dimensions are not necessarily on directly comparable scales. In order to align dimensions, the metric of each dimension can be transformed to reflect these across-dimension differences. To retain the measurement properties of the fitted Rasch model, we only consider linear metric transformations.

In this paper, we define aligned dimensions for the between-items multidimensional Rasch model for binary data. If dimensions are aligned and there are no missing data, then the This article is protected by copyright. All rights reserved. 
entire set of item difficulty parameters $\delta_{i(d)}$ are related to the observed proportion-correct scores $p_{i(d)}$ by a unique bijective function that does not depend on $d$. For this model, the $p_{i(d)}$ are sufficient statistics for the item parameters (Andersen, 1977), meaning that the estimated item parameters do not depend on any features of the data other than $p_{i(d)}$.

Therefore, dimension alignment ensures that the sufficient statistics imply the same item difficulty estimates regardless of dimension. As a result, items that have the same aligned difficulty estimate actually have the same operational characteristics, even if the items belong to different dimensions. Finally, because in the Rasch model, item and person parameters are on the same metric, if item difficulties are aligned to each other, then person parameters estimated from the aligned item parameters are also aligned.

There is no general closed-form expression for the functional relationship between $p_{i(d)}$ and $\delta_{i(d)}$ for the Rasch model. For a given population, the expected proportion of correct responses, denoted $E\left(p_{i(d)}\right)$, equals

$$
E\left(p_{i(d)}\right)=\int P_{i(d)}\left(\theta_{d}\right) g\left(\theta_{d}\right) \mathrm{d} \theta_{d},
$$

where $P_{i(d)}$ is defined in Equation (3) and $g\left(\theta_{d}\right)$ is the population distribution of person locations on dimension d.Importantly, Equation (7) shows that $p_{i(d)}$ depends not only on the difficulty of the item but on the distribution of scores on the dimension to which the item belongs. ${ }^{1}$ In the special case for which $g\left(\theta_{d}\right)$ is normal, Equation (7) is equivalent to the logistic-normal integral. Even in this simplified case, closed-form expressions of the logistic-normal integral are only approximations

${ }^{1}$ It is noteworthy that scale alignment methods do not necessarily result in equal latent trait variances across dimensions. Equation (7) shows that dimensions are necessarily aligned if the latent trait distribution is exactly the same across dimensions. However, it is not the case that matching the first two distributional moments across dimensions leads to alignment. For this reason, scaling methods that standardize scores on each dimension do not necessarily lead to aligned dimensions.

This article is protected by copyright. All rights reserved. 
(Demidenko, 2013). These approximations may be inaccurate even under normality, and may be even more inaccurate under non-normal trait distributions. Despite these caveats, we later demonstrate that the logistic function usually provides a fairly close approximation.

Dimension alighment requires solving for the $r_{d}$ and $s_{d}$ values used in Equations (4) - (6) for -

each dimension such that the $p_{i(d)}$ values are related to the transformed item parameters $\tilde{\delta}_{i(d)}$ by a bijective function. Below, we describe one previously developed method designed to align dimensions, delta-dimensional alignment (DDA), and propose a new method, logistic regression alignment (LRA). In the Appendix, we provide greater detail about how to implement these methods using Rasch modeling software.

As presented in this paper, scale alignment is an adjustment made to multidimensional item and person parameters that have been estimated using any identification constraints. In the future, it may be possible to implement alignment as an identification constraint (i.e., directly into the parameter estimation algorithm). For now, we recommend a multistage when applying scale alignment to real data. First, fit the multidimensional item response model. Second, apply either the DDA or LRA method to find the aligned item parameter estimates $\widetilde{\widehat{\boldsymbol{\delta}}}_{d}$ for each dimension. Third, anchor the $\widetilde{\widetilde{\boldsymbol{\delta}}}_{d}$ parameters to estimate person parameters or to fit a latent regression model. This multistage approach guarantees that the standard errors of person parameters are appropriate for the aligned dimensions.

\section{Method 1: Delta-Dimensional Alignment}

Although the delta-dimensional alignment (DDA) method has been used previously (as noted above), none of these applications give a detailed mathematical explanation of why the DDA method works, a description of how scale alignment affects the distribution of scores across dimensions, or a concrete definition of aligned dimensions. As it was originally This article is protected by copyright. All rights reserved. 
proposed, DDA assumed that the steepness parameters did not differ across dimensions, that

is, that all $\alpha_{d}$ equaled 1 both before and after alignment. As a result, dimension alignment (1)

using the original DDA method will generally lead to a worse-fitting model than a method that incorporates differing $\alpha_{d}$. Below, a modified DDA method is described that adjusts the dimension steepenesses and does not lead to any loss of model fit. Because the logic underlying the scale alignment process remains the same as that in the original DDA method, we will continue to refer to the method below as delta-dimensional alignment.

When scales are aligned, the proportions of correct responses $p_{i(d)}$ have a one-to-one relationship with the estimated item difficulties $\hat{\delta}_{i(d)}$, regardless of dimension. Furthermore, note that the $p_{i(d)}$ are the sufficient statistics for both the unidimensional and the multidimensional models, and that under the unidimensional model, the sufficient statistics will have a one-to-one relationship with the estimated item difficulties. Therefore, one way to obtain item diffieulties that are in the desired order is to fit all items to a unidimensional model. Because the unidimensional model will order the estimated item difficulties according to the order of the sufficient statistics, the means and variances of item parameters from the unidimensional model are an indication of the desired means and variances of item parameters in the multidimensional model.

To implement the DDA method, it is necessary to fit both a multidimensional model and a unidimensional model. Let $\mathcal{M}$ denote the multidimensional model, and let $\mathcal{U}$ denote the unidimensional model. For items belonging to each dimension $d$, the means and standard deviations of the multidimensional difficulty estimates $\widehat{\boldsymbol{\delta}}_{\mathcal{M} d}$ and of the unidimensional difficulty estimates $\widehat{\boldsymbol{\delta}}_{U d}$ are calculated. The DDA method then transforms the item difficulties for each dimension of the multidimensional model to have the same means and variances as

This article is protected by copyright. All rights reserved. 
the item difficulties for items on that dimension estimated relative to the unidimensional model. Put formally,

and

$$
\hat{r}_{d}=\frac{\operatorname{sd}\left(\widehat{\boldsymbol{\delta}}_{u d}\right)}{\operatorname{sd}\left(\widehat{\boldsymbol{\delta}}_{\mathcal{M} d}\right)}
$$

$$
\hat{s}_{d}=\operatorname{mn}\left(\widehat{\boldsymbol{\delta}}_{U d}\right)-\hat{r}_{d} \operatorname{mn}\left(\widehat{\boldsymbol{\delta}}_{\mathcal{M} d}\right)
$$

where $\operatorname{sd}(\boldsymbol{x})$ represents the standard deviation of $\boldsymbol{x}$, and $\operatorname{mn}(\boldsymbol{x})$ represents the arithmetic mean of $\boldsymbol{x}$. Equations (8) and (9) can be used to find $\hat{r}_{d}$ and $\hat{s}_{d}$ for each dimension. Those values can then be used in Equations (4) - (6) to find the transformed item and person parameters. Alternatively, one might choose only to transform the item parameters (Equations (4) and (6) and re-estimate person parameters conditional on the aligned item difficulties. Note that whenever the value of $\hat{r}_{d}$ differs across dimensions, the transformed steepnesses will also differ across dimensions.

Using the DDA method as defined in Equations (8) and (9) results in adjustments to each dimension based on information from the reference dimension. Instead, some users may find it more intuitive to preserve the metric of one of the dimensions and to transform the remaining dimensions. In this case, the same quantities can be used to calculate the DDA adjustment. Suppose that $d=1$ is specified as the unchanged dimension. Then, the appropriate transformation parameters for dimension $d$ are

$$
\hat{r}_{d}=\frac{\operatorname{sd}\left(\widehat{\boldsymbol{\delta}}_{u d}\right) \operatorname{sd}\left(\widehat{\boldsymbol{\delta}}_{\mathcal{M} 1}\right)}{\operatorname{sd}\left(\widehat{\boldsymbol{\delta}}_{\mathcal{M} d}\right) \operatorname{sd}\left(\widehat{\boldsymbol{\delta}}_{U 1}\right)},
$$

and

$$
\hat{S}_{d}=\frac{\operatorname{mn}\left(\widehat{\boldsymbol{\delta}}_{u d}\right)-\operatorname{mn}\left(\widehat{\boldsymbol{\delta}}_{u_{1}}\right)+\frac{\operatorname{sd}\left(\widehat{\boldsymbol{\delta}}_{\mathcal{U}_{1}}\right)}{\operatorname{sd}\left(\widehat{\boldsymbol{\delta}}_{\mathcal{M} 1}\right)} \operatorname{mn}\left(\widehat{\boldsymbol{\delta}}_{\mathcal{M} 1}\right)-\frac{\operatorname{sd}\left(\widehat{\boldsymbol{\delta}}_{u d}\right)}{\operatorname{sd}\left(\hat{\boldsymbol{\delta}}_{\mathcal{M} d}\right)} \operatorname{mn}\left(\widehat{\boldsymbol{\delta}}_{\mathcal{M} d}\right)}{\frac{\operatorname{sd}\left(\hat{\boldsymbol{\delta}}_{u_{1}}\right)}{\operatorname{sd}\left(\widehat{\boldsymbol{\delta}}_{\mathcal{M} 1}\right)}} .
$$

This article is protected by copyright. All rights reserved. 
Applying Equations (10) and (11) to $d=1$ gives $\hat{r}_{d}=1$ and $\hat{s}_{d}=0$, which defines an identity transformation in Equations (4) - (6). Any dimension may be chosen as the unchanged reference dimension. Different choices of reference dimension will lead to different aligned parameter estimates but will not affect the success of the DDA adjustment

(e.g., as assessed by the rank-order correlation between sufficient statistics and parameter estimates, dimensions combined).

Method 2: Logistic Regression Alignment

One disadyantage of the DDA method is that it requires fitting a unidimensional item response model in addition to the multidimensional model. Moreover, mean and variance statistics are often unstable, especially when dimensions are composed of only a few items. As such, the DDA method might be overly sensitive to outliers or to estimated item parameters with large standard errors. Responding to these concerns, another method for scale alignment is developed below that builds directly on the above definition of aligned dimensions.

Recall that there is no general closed-form solution for the relationship between $p_{i(d)}$ and $\delta_{i(d)}$ in Equation 7. However, we have found that simple logistic regression often provides a highly accurate description of the relationship between sufficient statistics estimated item difficulties under Rasch models for binary data. For example, in the math and science data set described below under "Illustration", the root mean squared difference (RMSD) between the model-predicted $p$ and the observed $p$ ranged from .0004 to .0006 (on a 0-1 probability metric). Moreover, across all of the fitted logistic regression models for data simulated under the two simulation studies described below, the median RMSD equaled .0012 and the maximum RMSD equaled .0083 . Because the logistic regression approximation 


\section{SCALE ALIGNMENT}

usually describes the data very well, the intercept $\hat{\gamma}_{0 d}$ and slope $\hat{\gamma}_{1 d}$ of the logistic regression of $p_{i(d)}$ on $\hat{\delta}_{i(d)}$ for dimension $d$ can be used to align dimensions by placing estimated item difficulties onto the same logistic regression curve.

The LRA technique hinges on the logistic approximation to the relationship between $p_{i(d)}$ and $\delta_{i(d)}$. To implement the LRA method, first fit a between-items multidimensional model. Then, for each dimension, calculate $\hat{\gamma}_{0 d}$ and $\hat{\gamma}_{1 d}$ from the logistic regression of the observed responses to item $i$ (the average of which equals $p_{i(d)}$ ) on $\hat{\delta}_{i(d)}$ :

$$
\operatorname{logit}\left(\operatorname{Pr}\left(y_{i j}=1 \mid \hat{\delta}_{i(d)}\right)\right)=\hat{\gamma}_{0 d}+\hat{\gamma}_{1 d} \hat{\delta}_{i(d)}
$$

where $\operatorname{Pr}\left(y_{i j}=1 \mid \hat{\delta}_{i(d)}\right)$ is the probability that person $j$ responds correctly to item $i$ conditional on the (previously computed) multidimensional difficulty estimate for item $i$. Next, choose a dimension for which the item difficulties will not be transformed (throughout this section, $d=1$ denotes the unchanged dimension and hence $\hat{r}_{1}=1$ and $\left.\hat{s}_{1}=0\right)$. The scale transformation parameters $\hat{r}_{d}$ and $\hat{s}_{d}$ can then be found by

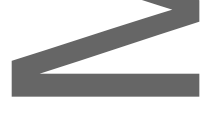

$$
\hat{r}_{d}=\frac{\hat{\gamma}_{1 d}}{\hat{\gamma}_{11}}
$$

and

$$
\hat{s}_{d}=\frac{\hat{\gamma}_{0 d}-\hat{\gamma}_{01}}{\hat{\gamma}_{11}}
$$

\footnotetext{
In practice, we recommend that both DDA and LRA be applied to the same data.
}

Researchers can then choose the solution that objectively provides better alignment. Because alignment is defined in terms of the relationship between sufficient statistics and parameter estimates, the absolute rank-order correlation between these two quantities (dimensions combined) is an appropriate indicator of the success of either alignment method. In a later section, we describe simulation studies that show few systematic advantages of either method This article is protected by copyright. All rights reserved. 


\section{SCALE ALIGNMENT}

over the other in terms of this criterion.

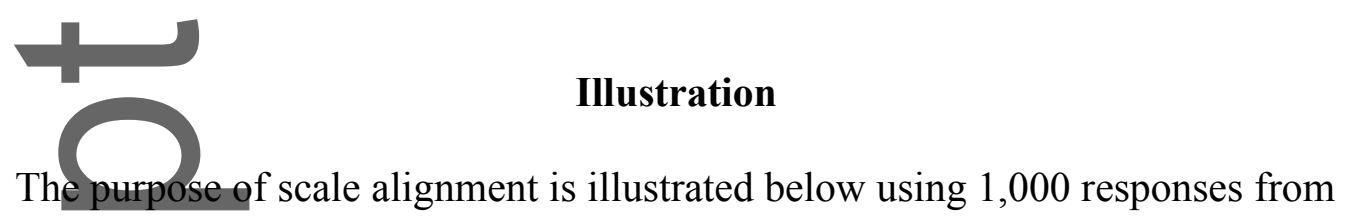
Australianstudents to 12 multiple-choice TIMSS items. This data set is described in chapters 3 and 9 of the ConQuest manual (Wu, Adams, Wilson, \& Haldane, 2007), and it is available within the ConQuest tutorial materials. In this data set, each item was scored dichotomously as correct or incorrect, and missing responses were scored as incorrect. The first six items measure mathematics ability and the final six items measure science ability.

A two-dimensional Rasch model for binary item responses was fit to these data using ConQuest. The model was fit twice, first by setting equal to 0 the mean ability on each dimension, and second by setting equal to 0 the mean item difficulty on each dimension. In Figure 3, the estimated item difficulties are plotted against the proportion-correct scores. To aid comparisons, the estimated item difficulties are connected by a best-fit logistic regression curve for each dimension. The left-side panel corresponds to the model for which mean person abitities were constrained to equal 0 , and the right-side panel corresponds to the model for which mean item difficulties were constrained equal to 0 . In addition, two items are highlighted, one on each dimension. In terms of the observed proportion-correct, the highlighted mathematics item is slightly more difficult than the highlighted science item, $p_{\text {math }}=.765$ and $p_{\text {science }}=.767$. However, both fitted models suggest that the mathematics item is easier than the science test item. That is, when constraining the person mean, $\hat{\delta}_{\text {math }}=$ -1.46 and $\hat{\delta}_{\text {science }}=-1.38$, and when constraining the item mean, $\hat{\delta}_{\text {math }}=-.66$ and $\hat{\delta}_{\text {science }}=-.01$. Because the metrics for math and science are separately defined, this 
result should not be surprising. However, there is no reason why the estimated item

difficulties cannot be aligned, and dimension alignment provides a clear basis for which to compare item or person scores across dimensions.

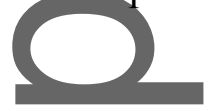

The effects of scale alignment for the example data set are illustrated in Figure 4. This figure shows the results using both the DDA and LRA adjustments (both with an unchanged first dimension) to align dimensions that were estimated by constraining the person mean equal to zero. In Panels A and B, the success of the DDA and LRA alignment methods is evident because the DDA-adjusted $\hat{\delta}$ and LRA-adjusted $\hat{\delta}$ lie on the same estimated logistic regression curve regardless of dimension. In other words, the relationship between the math and science items is correctly shown in Panels A and B of Figure 4 because the two methods have been brought into alignment. In contrast, Panels $C$ and D illustrate that scale alignment using DDA or LRA does not align the person sufficient statistics with the person parameter estimates. This is as it should be. Because it is usually implausible that tests are equally difficult, it should not necessarily be the case that a subject who obtains the same proportioncorrect score on both subtests should have the same estimated ability on the two dimensions. In fact, for both methods, the difference between WLEs on the two dimensions appears to be related to the mean difference in item difficulty on the two dimensions. Specifically, the mean difference in aligned item difficulties across dimensions equals .66 for the DDA alignment and .67 for the LRA alignment. Similarly, the across-dimension mean differences in WLEs conditional on person sufficient statistics equals .66 for both the DDA and LRA alignment methods. This article is protected by copyright. All rights reserved. 


\section{Simulation Studies}

As the above illustration demonstrated, the DDA and LRA methods effectively align dimensions in the example data set. Next, two simulation studies were designed to evaluate the characteristics and performance of these two alignment methods. These simulations were designed to investigate several scale alignment issues simultaneously. A primary goal was to determine whether scale alignment using DDA or LRA effectively aligns dimensions in a wide variety of contexts. A secondary goal was to establish whether any major performance differences exist between the two scale alignment methods. A final goal was to investigate patterns of estimated $r_{d}$ and $s_{d}$ alignment parameters to identify contexts in which scale alignment has the greatest effect.

Simulation 1

Simulation 1 aimed to establish (a) which factors affect the accuracy of scale alignment, and (b) whether there are any performance differences between DDA and LRA. In particular, the extents to which sample size, test length, the distribution of person parameters, and the correlation between dimensions affect scale transformations were investigated. In all cases, two-dimensional binary item response data were simulated with between-items multidimensionality. For all simulations, the latent trait distribution of Dimension 1 and the distribution of item difficulties for both dimensions were held constant. Specifically, person abilities on Dimension 1 and the item difficulties on Dimensions 1 and 2 were all generated from standard normal distributions. The data-generating $\theta_{1}$ values and item difficulties were redrawn for each data set.

The following factors were manipulated. Sample size was set equal to either 100, 200, 500 , or 1000 . Four combinations of subtest lengths were simulated by specifying Dimensions This article is protected by copyright. All rights reserved. 
SCALE ALIGNMENT

1 and 2 to have either 5 or 20 items. In addition, the mean of $\theta_{2}$ was set equal to either $0, .5$, or 1 . The standard deviation of $\theta_{2}$ was set equal to either $.5,1,1.5$, or 2 . The skewness of $\theta_{2}$ equaled either 0 or .75 , and the kurtosis of $\theta_{2}$ equaled either 0 or 3 . Finally, the correlation between $\theta_{1}$ and $\theta_{2}$ was set equal to $0, .3, .6$, or 9 . All manipulated factors were fully crossed for a total of 3072 conditions $(4$ sample sizes $\times 2$ Dimenesion 1 test lengths $\times 2$ Dimension 2 test lengths $\times 3$ mean $\theta_{2}$ values $\times 4$ standard deviations of $\theta_{2} \times 2$ skewness values of $\theta_{2} \times 2$ kurtosis values of $\theta_{2} \times 4$ correlations between $\theta_{1}$ and $\theta_{2}$ ). We conducted 20 replications of each simulated condition.

After the data were generated, multidimensional item parameters were estimated using the TAM (Robitzsch, et al., 2017) package for R (R Core Team, 2018) and by constraining the mean $\theta$ on each dimension equal to zero. Multidimensional latent trait values were generating according to the specified correlation, mean, standard deviation, skewness, and kurtosis values using the method developed by Vale and Maurelli (1983) as implemented in the SimDesign (Chalmers, 2017) package for R. Using these randomly generated latent trait values and item difficulties, multivariate binary item response data were generated using the TAM (Robitzsch et al., 2017) package. After fitting the data to multidimensional models, all items were fit to a unidimensional model (for use in the DDA method) using TAM, constraining person means to zero for both dimensions. The DDA alignment was performed using Equations (10) and (11) such that $\hat{r}_{1}=1$ and $\hat{s}_{1}=0$. For the LRA method, the logistic regression curves were estimated using the glm function included in the R stats library. As with DDA, the LRA Dimension 1 was unchanged such that that $\hat{r}_{1}=1$ and $\hat{s}_{1}=0$.

The accuracy of the DDA and LRA alignment procedures was assessed using Kendall's (1970) $\tau$ coefficient for rank-order correlations. Specifically, combining dimensions, rank- 
order correlations were calculated between the item sufficient statistics and the aligned item difficulty estimates. Throughout this paper, the absolute values of these rank-order correlations are reported because there is always an inverse relationship between $p_{i(d)}$ and $\delta_{i(d)}$ (more difficult items will have lower proportions of correct responses). The median absolute rank-order correlation between $p$ and $\tilde{\hat{\delta}}$ across all conditions equaled 1 for both LRA and DDA. When evaluating the magnitude of the alignment effect, it is worthwhile to compare these correlations to the rank-order correlations between the item sufficient statistics and the unaligned item difficulty estimates. Because within dimensions, this rank-order correlation should equal 1, the across-dimension correlation should also be rather large for the unaligned parameters. Before alignment, the median rank-order correlation between $p$ and $\hat{\delta}$ equaled .987 , and the DDA and LRA alignment methods provided a median .013 and .010 increase in absolute rank-order correlation. In addition, both DDA and LRA led to aligned rank-order correlations equal to 1.000 in $55 \%$ of fitted models, whereas before alignment only $23 \%$ of fitted models had absolute rank-order correlations of 1.000 .

We next investigated the conditions for which scale alignment has the greatest potential effect, that is, which conditions had the lowest absolute rank-order correlation between $p$ and $\hat{\delta}$ before aligmment. We regressed these absolute rank-order correlations on all manipulated factors and interactions, which resulted in a multiple $R^{2}$ of only .38 . The greatest effect size was found for the main effect of the standard deviation of $\theta_{2}\left(\eta^{2}=.27\right)$, followed by the interaction of the two test lengths $\left(\eta^{2}=.04\right)$, the three-way interaction of the two test lengths with the standard deviation of $\theta_{2}\left(\eta^{2}=.02\right)$, the interaction between the standard deviation and kurtosis of $\theta_{2}\left(\eta^{2}=.009\right)$, and the main effect of the kurtosis of $\theta_{2}\left(\eta^{2}=\right.$ .007). Part of the reason why test length has a relatively large effect is that with the 5-item This article is protected by copyright. All rights reserved. 
conditions, there are only a small number of rank-order correlations that can be observed, and so the rank-order correlation is more sensitive to a single deviation from perfect ordering. Indeed, among the conditions with only 5 items per dimension, the minimum absolute rankorder correlation equaled .64 whereas among the conditions with 20 items in at least one dimension, the minimum absolute rank-order correlation equaled .87. Apart from sample size, the most salient factors relate to the distribution of $\theta_{2}$, and in particular the standard deviation of $\theta_{2}$. Further investigation suggests that the smallest absolute rank-order correlations occur when the standard deviation of $\theta_{2}$ is not equal to 1 (i.e., not equal to the standard deviation of $\theta_{1}$ ) — the median absolute rank-order correlation for $\theta_{2}=\{0.5,1.0,1.5,2.0\}$ equals $.983, .996, .982$, and .961 , respectively. These results suggest that scale alignment is most needed when the latent variable variances on the two dimensions differ.

We next considered whether the accuracy of DDA or LRA, as evaluated by the absolute rank order correlation between $p$ and $\tilde{\hat{\delta}}$, is affected by any of the manipulated factors. In general, it was found that the accuracy of DDA or LRA procedures could not be predicted based on the manipulated factors. Specifically, when the absolute rank-order correlations between $p$ and $\tilde{\hat{\delta}}$ were regressed on all of the manipulated factors and their interactions, the multiple $R^{2}$ was only .20 for the DDA adjustment and .17 for the LRA adjustment. These results indicate that most of the variability in the performance of the DDA and LRA methods is not associated with the factors manipulated in this first study. Although there is some systematic variability in the rank-order correlations between $p$ and $\tilde{\hat{\delta}}$ before alignment, the DDA and methods may be used equally well to achieve alignment in a wide variety of data sets.

This article is protected by copyright. All rights reserved. 
Finally, we compared the performance of the DDA and LRA methods, and found that these methods usually led to very similar solutions. In $97.2 \%$ of replications, the two alignment methods resulted in exactly the same rank-order correlation between $p$ and $\tilde{\hat{\delta}}$, in $1.9 \%$ of replications DDA led to a higher absolute rank-order correlation than LRA, and in 口 $.9 \%$ of replications LRA led to a higher rank-order correlation than DDA. The Pearson product-moment correlation equaled .999 between the DDA $\hat{r}_{2}$ and the LRA $\hat{r}_{2}$, and equaled .962 between the DDA $\hat{s}_{2}$ and the LRA $\hat{s}_{2}$. Similarly, the DDA and LRA methods resulted in similar rank-order correlations between $p$ and $\tilde{\hat{\delta}}$; the root mean squared difference between these two sets of rank-order correlations equaled .002 across the studied conditions. In a small number of eases, there were large differences $(>.02)$ in the rank-order correlations recovered by the DDA and LRA methods. All of these large differences occurred when both dimensions included only 5 items. Notably, in practice it is always possible to compute both the DDA and LRA alignments and to empirically compare their performance using a rankorder correlation between $p$ and $\tilde{\hat{\delta}}$.

Overall, Simulation 1 provides initial evidence that (a) both DDA and LRA are broadly effective in aligning the dimensions of a multidimensional Rasch model when the model is correctly specified, (b) characteristics such as sample size, test length, the distribution of $\theta_{2}$, and the correlation between dimensions have little effect on the effectiveness of the alignment procedures, and (c) there are no major differences in performance between DDA and LRA.

\section{Simulation 2}

A second simulation study was conducted to evaluate the patterns of $\hat{r}_{2}$ and $\hat{s}_{2}$ under more controlled conditions. Based on the results of Simulation 1 and the theoretical results presented after Equation (7), we decided to focus Simulation 2 on the effects of the latent trait This article is protected by copyright. All rights reserved. 
SCALE ALIGNMENT

distribution. In Simulation 2, binary item response data were generated under a twodimensional Rasch model with between-items multidimensionality. Data were generated for 1000 examinees and 10 items on each dimension. Both dimensions included the same set of data-generating item difficulties evenly spaced between -2 and 2 . In these simulations, the correlation between dimensions equaled .5 , and the $\theta_{1}$ values were generated from a standard normal distribution. The only manipulated parameters in Simulation 2 were those that defined the $\theta_{2}$ distribution. Namely, the mean of $\theta_{2} \in\{0, .5,1,1.5,2\}$, the standard deviation of $\theta_{2} \in\{.5,1,1.5,2\}$, the skewness of $\theta_{2} \in\{0, .5,1,1.5,2\}$, and the kurtosis of $\theta_{2} \in$ $\{-2,-1,0,1,2,3\}$. These four factors were fully crossed, except for those skewness/kurtosis combinations that are impossible (Fleishman, 1978), for a total of 260 conditions. Each condition was replicated 100 times. As in Simulation 1, the method of Vale and Maurelli (1983) was used to simulate multivariate normal and non-normal data using the SimDesign package (Chalmers, 2017) for R (R Core Team, 2018). After the data were generated, between-items multidimensional models were estimated and aligned item difficulty estimates with DDA and LRA using the same methodological choices described for Simulation 1.

As was found in Simulation 1, both the DDA and LRA methods successfully aligned scales in nearly all conditions and replications. Both methods had median absolute rank-order correlations of 1.000 between $p$ and $\tilde{\hat{\delta}}$. The minimum absolute rank-order correlation equaled .956 for DDA and .968 for LRA. Compared to the unaligned parameters, both DDA (1) and LRA had a median increase in absolute rank-order correlations of .024. Moreover, none of the manipulated conditions (i.e., distributions of $\theta_{2}$ ) resulted in systematically lower rankorder correlations for either DDA or LRA. When the rank-order correlations were regressed on all manipulated factors and their interactions, the resulting $R^{2}$ equaled .04 for DDA and 


\section{SCALE ALIGNMENT}

.07 for LRA. These results support the Simulation 1 results that suggest the DDA and LRA methods can be applied to align multidimensional Rasch scales in a broad variety of contexts.

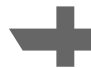

Next, the patterns of $\hat{r}_{2}$ and $\hat{s}_{2}$ values obtained by DDA and LRA were investigated.

Recall that for both methods $\hat{r}_{1}=1$ and $\hat{s}_{1}=0$, and so $\hat{r}_{2}=1$ and $\hat{s}_{2}=0$ would define an identity (no change) transformation for Dimension 2. For the conditions manipulated in Simulation 2, it was found that $.67 \leq \hat{r}_{2} \leq 1.20$ and $-.07 \leq \hat{s}_{2} \leq .04$ for DDA and that $.66 \leq \hat{r}_{2} \leq 1.23$ and $-.08 \leq \hat{s}_{2} \leq .03$ for LRA. It was also found that DDA and LRA reliably suggest similar metric transformations. The Pearson product-moment correlation between the DDA and LRA $\hat{r}_{2}$ equaled .998 and between the DDA and LRA $\hat{s}_{2}$ equaled .814 . Moreover, the root mean squared difference between $\hat{r}_{2, D D A}$ and $\hat{r}_{2, L R A}$ equaled .013 and between $s_{2, D D A}$ and $s_{2, L R A}$ equaled .009. These results show that although the two methods find very similar solutions on average, there are some small differences between the two methods. Upon closer inspection, we found that LRA usually suggests $\hat{r}_{2}$ values further from 1 than does DDA. Moreover, LRA usually suggests larger shifts (in terms of absolute value of $\hat{S}_{2}$ ) than does DDA. Despite these differences, it should be emphasized that the LRA and DDA methods provide, in the vast majority of conditions, equally accurate alignments.

Next, the across-replication variability of $\hat{r}_{2}$ and $\hat{s}_{2}$ for the DDA and LRA methods was considered. Specifically, for each method and for each of the 260 experimental conditions, the standard deviation of $\hat{r}_{2}$ and $\hat{s}_{2}$ across the 100 replications was computed. In all but one condition, the DDA $\hat{r}_{2}$ was less variable across replications than the LRA $\hat{r}_{2}$, and in $89 \%$ of conditions, the DDA $\hat{s}_{2}$ was less variable across replications than the LRA $\hat{s}_{2}$. In the one condition where the LRA $\hat{r}_{2}$ was less variable than the DDA $\hat{r}_{2}$ involved a $\theta_{2}$ mean of 0 , standard deviation of 2 , skewness of .5 , and kurtosis of 1 . In contrast, in all cases for which 
SCALE ALIGNMENT

the DDA $\hat{s}_{2}$ was always less variable than the LRA $\hat{s}_{2}$, the mean $\theta_{2}$ equaled 1.5 or 2.0 and the standard deviation of $\theta_{2}$ equaled .5 or 1 . Note that these conditions are those for which Dimension 2 should be least reliably determined. Specifically, when the mean of $\theta_{2}$ is far from 0 and the standard deviation of $\theta_{2}$ is relatively small, the $\theta_{2}$ distribution is less wellmatched the item difficulty distribution. Thus, one interpretation of these results is that the DDA alignment is more stable than the LRA alignment except when the second dimension is not estimated reliably. Nevertheless, the differences in the stability of the alignment coefficients are very small and appear to have little effect on the accuracy of the alignment.

Finally, we explored whether characteristics of the $\theta_{2}$ distribution predict $\hat{r}_{2}$ and $\hat{s}_{2}$. Box plots of these exploratory results are shown for $\hat{r}_{2}$ in Figure 5, and for $\hat{s}_{2}$ in Figure 6 . Because DDA and LRA lead to very similar solutions, and because DDA is usually less variable than LRA, these plots only show the DDA results. The plots in Figures 5 and 6 are conditional on onty the standard deviation, skewness, and kurtosis of $\theta_{2}$ because the mean of $\theta_{2}$ predicts only $2 \%$ of the variance in the DDA $\hat{s}_{2}$ and only $.4 \%$ of the variance in the DDA $\hat{r}_{2}$.

For these simulations, it was found that the standard deviation of $\theta_{2}$ is highly predictive of $\hat{r}_{2}$. For DDA, the $R^{2}$ value from regressing $\hat{r}_{2}$ on $s d\left(\theta_{2}\right)$ equals .95 , and for LRA, this $R^{2}$ value equals .94. Recall that when the distribution of $\theta_{1}$ is identical to the distribution of $\theta_{2}$, no scale realignment is necessary. Thus, when $m n\left(\theta_{2}\right)=0, \operatorname{sd}\left(\theta_{2}\right)=$ $1, \operatorname{skew}\left(\theta_{2}\right)=0$, and $\operatorname{kurt}\left(\theta_{2}\right)=0$, the expected scale transformation parameters are $\hat{r}_{2}=$ 1 and $\hat{s}_{2}=0$. For the 100 replications for which the identity transformation is appropriate, the mean $\hat{r}_{2}=.998$ and the mean $\hat{s}_{2}=.000$ for both DDA and LRA. The standard deviations of $\hat{r}_{2}$ and $\hat{s}_{2}$ values equaled .016 and .001 for both DDA and LRA. These results This article is protected by copyright. All rights reserved. 
confirm that both the DDA and LRA methods suggest transformations close to identity when the identity transformation is expected.

As the conditioning plot in Figure 5 shows, the standard deviation of $\theta_{2}$ is inversely

related to $\hat{r}_{2}$. As expected from the facts that the standard deviation of $\theta_{1}=1$ and $\hat{r}_{1}=1, \hat{r}_{2}$ is closest to 1 when the standard deviation of $\theta_{2}=1$. This result implies that, when the standard deviation of $\theta_{2}$ is larger than the standard deviation of $\theta_{1}$ (before alignment), then the aligned Dimension 2 will also have higher steepnesses $\left(\tilde{\alpha}_{2}\right)$ than Dimension 1 after alignment. In addition, the skewness and kurtosis of $\theta_{2}$ affect the DDA $\hat{r}_{2}$. Specifically, Figure 5 shows that larger kurtosis values lead to higher $\hat{r}_{2}$ values, especially when the standard deviation of $\theta_{2}$ is greater than 1 . Finally, the skewness of $\theta_{2}$ affects the variability of $\hat{r}_{2}$. This result might be explained in terms of the reliability of Dimension 2 . When Dimension 2 is less reliably estimated, as is the case here as the $\theta_{2}$ distribution becomes increasingly skewed, then this lack of reliability leads to increased variability in the estimated scale transformation coefficients.

The effects of the standard deviation, skewness, and kurtosis of $\theta_{2}$ on the distribution of the DDA $\hat{s}_{2}$, as shown in Figure 6, are more complex than for the DDA $\hat{r}_{2}$. First note that relatively small shifts are estimated when $s d\left(\theta_{2}\right)=1$. Conditional on $s d\left(\theta_{2}\right)=1$, the $\hat{s}_{2}$ values are uniformly close to 0 and are little affected by the skewness and kurtosis of $\theta_{2}$ (though somewhat more variable with low kurtosis). Similarly, when $s d\left(\theta_{2}\right)=.5$, the estimated shift parameters tend to be slightly greater than 0 and insensitive to the skewness and kurtosis values. In contrast, when the standard deviation of $\theta_{2}$ is greater than 1 , the estimated shift parameters tend to be negative, especially for large amounts of skewness and low kurtosis. These conditions lead to $\hat{s}_{2}$ values that are lower and also more variable than in This article is protected by copyright. All rights reserved. 
the other conditions. As with $\hat{r}_{2}$, it appears that this latter result is confounded with the reliability of Dimension 2. Finally, it should be noted that the $\hat{s}_{2}$ values are very close to zero for most of these conditions because models were fit by constraining the means of $\theta_{1}$ and $\theta_{2}$ equal to zero. If instead the item difficulty means were set equal to zero, the estimated shifts would be of greater magnitude, and would largely reflect differences in the person distribution means. Returning to Equation (7), we see that the only mediating factor affecting the relationship between the item sufficient statistics and the item difficulties is the distribution of the latent trait. Thus, in a multidimensional model, if the distribution of person scores is identical for each dimension, then no realignment is necessary. In this case, constraining person means equal to zero accomplishes much of the work of scale alignment. However, as the real and simulated examples given in this paper show, the DDA and LRA methods often suggest scale transformations that are reliably different than the identity transformation

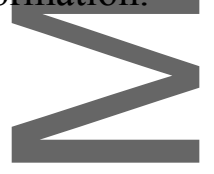

\section{Discussion}

When several related but qualitatively distinct constructs are assessed simultaneously (e.g., verbalability and mathematics ability), it is desirable to report these scores on scales that facilitate direct comparisons (Angoff, 1971; Holland \& Dorans, 2006; Kelley, 1923). However, in multidimensional Rasch analyses, scores estimated on different dimensions are not necessarily on comparable scales. This feature of multidimensional models has been identified in the past, and DDA (Schwartz \& Ayers, 2011; Yamada et al., 2006) has been proposed as one alignment method. Because the DDA method requires the extra computational cost of fitting additional item response models, the LRA alignment method is developed in this paper. Through a series of real and simulated data examples, is it shown that 
both DDA and LRA are effective alignment methods for a wide variety of datasets. In practice, we recommend applying both the DDA and LRA methods and using whichever provides the highest absolute rank-order correlation between the item sufficient statistics and the aligned item parameter estimates.

The concept of scale alignment provides an important link between classical test theorybased battery scaling methods (Angoff, 1971) and item response theory. Rather than rescaling dimensions according to the full distribution of scores, IRT-based scale alignment transforms dimensions so that an individual's score estimates are directly comparable across dimensions. Because IRT-based scale alignment relates sample statistics to parameter estimates, it is inherently a sample-specific procedure (as is battery scaling). If aligned item parameters are applied to new population, there is no necessary relationship between the new sample statistics and the item difficulties. However, if the scales are aligned with a large representative sample from the population of examinees, it is expected that the alignment properties will translate to other representative samples from that population.

There are several issues involved in scale alignment that are not addressed in this paper. Sufficient statistics are not available in the presence of missing data, and so additional research needs to be done to investigate the usefulness of alignment methods when some data are missing. Furthermore, because the definition of alignment used in this paper depends on sufficient statistics, these ideas do not apply directly to IRT models with no available sufficient statistics (i.e., models outside the Rasch family such as the two- and threeparameter models). Finally, the extension of the DDA and LRA methods to polytomous data does not follow directly from these results, primarily because the sufficient statistics in the partial credit model do not have a monotonic relationship with the estimated item parameters (Masters, 1982, 1988). We plan to develop methods for scale alignment for polytomous data This article is protected by copyright. All rights reserved. 
SCALE ALIGNMENT

in a separate paper.

In this paper, scale alignment in multidimensional Rasch models is formally defined, and two methods are described for aligning dimensions. After applying these methods to real and simulated data sets, it was shown that the two alignment methods lead to similar results and are both broadly effective. It is recommended that scale alignment be routinely used in between-items multidimensional Rasch models so that the scores for individual and groups can be appropriately compared across dimensions.

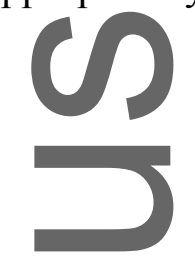

\section{References}

Ackerman, T. A. (1988, April). An exploration of differential item functioning from a multidimensional perspective. Paper presented at the Annual Meeting of the American Educational Research Association, New Orleans.

Ackerman, T. A. (1992). A didactic explanation of item bias, item impact, and item validity from a multidimensional perspective. Journal of Educational Measurement, 29, 67-

91.

Adams, R. J., Wilson, M., \& Wang, W.-C. (1997). The multidimensional random coefficients multinomial logit model. Applied Psychological Measurement, 21, 1-23.

Andersen, E. B. (1977). Sufficient statistics and latent trait models. Psychometrika, 42, 69-81.

Angoff, W.H. (1971). Scales, norms, and equivalent scores. In R. L. Thorndike (Ed.). Educational measurement (2 ${ }^{\text {nd }}$ ed.) (pp. 508-600). Washington, DC: American Council of Education.

Bolsinova, M., Maris, G., \& Hoijtink, H. (2016) Unmixing Rasch scales: How to score an educational test. The Annals of Applied Statistics, 10, 925-945.

This article is protected by copyright. All rights reserved. 
SCALE ALIGNMENT

Castellano, K. E., Duckor, B., Wihardini, D., Tellez, K., \& Wilson, M. (2016, Winter).

Assessing academic language in an elementary mathematics teacher licensure exam.

Teacher Education Quarterly.

Chalmers, P. (2017). SimDesign: Structure for Organizing Monte Carlo Simulation Designs.

R package version 1.7 https://CRAN.R-project.org/package=SimDesign

Davey, T., Oshima, T. C., \& Lee, K. (1996). Linking multidimensional item calibrations.

Applied Psychological Measurement, 20, 405-416.

Davey, T., Oshima, T. C., \& Lee, K. (2000). Multidimensional linking: Four practical approaches. Journal of Educational Measurement, 37, 357-373.

Dorans, N. J. (2002). Recentering and realigning the SAT score distributions: How and why. Joutrnal of Educational Measurement, 39, 59-84.

Flanagan, J.C. (1951). Units, scores, and norms. In E. F. Lindquist (Ed.), Educational measurement (pp. 695-763). Washington, DC: National Academy Press.

Fleishman, A. P.(1978). A method for simulating non-normal distributions. Psychometrika, $43,521-532$.

Gochyyev, (2015). Essays in psychometrics and behavioral statistics. Unpublished doctoral dissertation.

Hoijtink, H., Rooks, G., \& Wilmink, F. W. (1999). Confirmatory factor analysis of items with a dichotomous response format using the multidimensional Rasch model.

Psychological Methods, 4, 300-314.

Holland, P. W., \& Dorans, N. J. (2006). Linking and equating. In R. L. Brennan (Ed.) Educational measurement (4th ed. pp. 187-220). Westport, CT: American Council on Education and Praeger.

Kendall, M. G. (1970). Rank correlation methods. London: Griffin.

This article is protected by copyright. All rights reserved. 
Kelley, T. L. (1923). A new method for determining the significance of differences in intelligence and achievement scores. Journal of Educational Psychology, 14, 321-333. Masters, G. N.(1982). A Rasch model for partial credit scoring. Psychometrika, 47, 149-174. Masters, G. N. (1988). The analysis of partial credit scoring. Applied Measurement in Education, 1, 279-297.

McKinley, R. L., \& Reckase, M. D. (1983). An extension of the two-parameter logistic model to the multidimensional latent space (Research Report ONR 83-2). Iowa City, IA: The American College Testing Program.

Morell, L., Collier, T., Black, P., \& Wilson, M. (2017). A construct-modeling approach to develop a learning progression of how students understand the structure of matter. Journal of Research in Science Teaching, 54, 1024-1048.

Osborne, J.F., Henderson, J. B., MacPherson, A., Szu, E., Wild, A., \& Yao, S.-Y. (2016). The development and validation of a learning progression for argumentation in science. Journat of Research in Science Teaching, 53, 821-846.

R Core Team. (2018). R: A language and environment for statistical computing [Computer software]. Vienna, Austria. Available from http://www.R-project.org/

Robitzsch, A., Kiefer, T., \& Wu, M. (2017). TAM: Test analysis modules. R package version 2.6-2. https://CRAN.R-project.org/package $=$ TAM

Schwartz, R. A. (2012). The development and psychometric modeling of an embedded assessment for a data modeling and statistical reasoning learning progression Unpublished doctoral dissertation.

Schwartz, R., \& Ayers, E. (2011). Delta dimensional alignment: Comparing performances across dimensions of the learning progression for assessing modeling and statistical 
SCALE ALIGNMENT

reasoning. Berkeley, CA: Unpublished manuscript, University of California, Berkeley.

Thurstone, L. L. (1935). The vectors of the mind. Chicago, IL: University of Chicago Press.

Twiss, J., \& McKenna, S. P. (2015). Comparing the impact of psoriasis and atopic dermatitis on quality of life: Co-calibration of the PSORIQoL and QoLIAD. Quality of Life

Research, 24, 105-113.

Vale, C., \& Maurelli, V. (1983). Simulating multivariate nonnormal distributions.

Psychometrika, 48, 465-471.

Wang, M. (1986, April). Fitting a unidimensional model to multidimensional item response

data. Paper presented at the Conference of the Office of Naval Research Contractors, Gatlinburg, TN.

Whitely, S. E. (1980). Multicomponent latent trait models for ability tests. Psychometrika, 45, $479-494$.

Wu, M., Adams, R., Wilson, M., \& Haldane, S. (2007). ACER Conquest: Generalised item response modelling software (Version 2.0) [computer software]. Melbourne, Australia: ACER.

Yamada, H., Draney, K., Karelitz, T., Moore, S., \& Wilson, M. (2006). Comparison of dimension-aligning techniques in a multidimensional IRT context. Presented at the $13^{\text {th }}$ International Objective Measurement Workshop, Berkeley, CA.

Yao, S.-Y., Wilson, M., Henderson, J. B., \& Osborne, J. (2015). Investigating the function of content and argumentation items in a science test: A multidimensional approach.

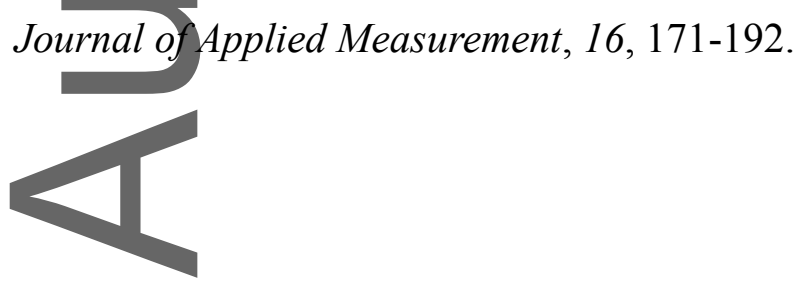

This article is protected by copyright. All rights reserved. 


\section{Appendix}

Special care must be taken when anchoring the $\widetilde{\widetilde{\boldsymbol{\delta}}}_{d}$ parameters in some Rasch modeling software such as ConQuest (Wu, Adams, Wilson, \& Haldane, 2007). Specifically, some item response modeling software expresses the multidimensional Rasch model as

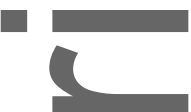

$$
\operatorname{logit}\left(P_{i(d) j}\right)=\alpha_{d} \theta_{d j}+\xi_{i(d)}
$$

instead of the parameterization used throughout the paper:

$$
10 \operatorname{logit}\left(P_{i(d) j}\right)=\alpha_{d}\left(\theta_{d j}-\delta_{i(d)}\right) \text {. }
$$

Note that scale alignment methods align the $\hat{\delta}_{i(d)}$ values rather than the $\hat{\xi}_{i(d)}$ values, and that the two values will differ by more than a sign when $\alpha_{d} \neq 1$. Thus, when using software that uses the parameterization in Equation (A1), further adjustments must be made to align the $\hat{\xi}_{i(d)}$ parameters. Specifically,

$$
\tilde{\hat{\xi}}_{i(d)}=\hat{\xi}_{i(d)}-\frac{\alpha_{d} s_{d}}{r_{d}},
$$

and no further adjustments are needed for the aligned steepnesses $\tilde{\alpha}_{d}$.

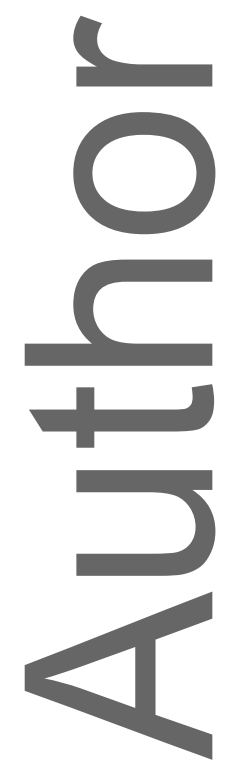

This article is protected by copyright. All rights reserved. 


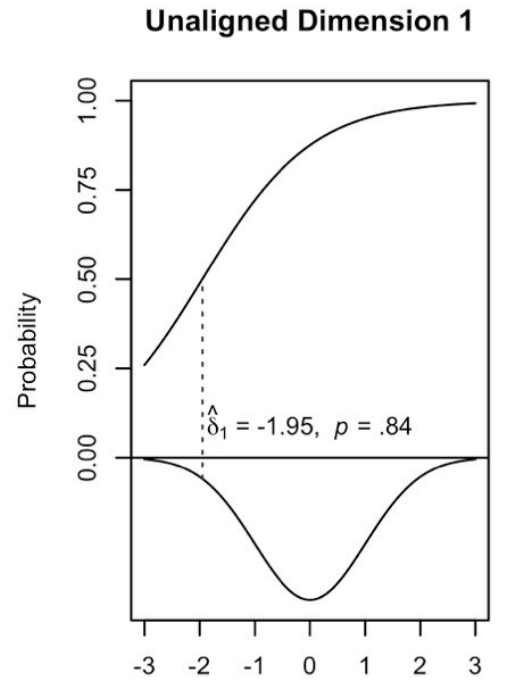

$\theta_{1}$

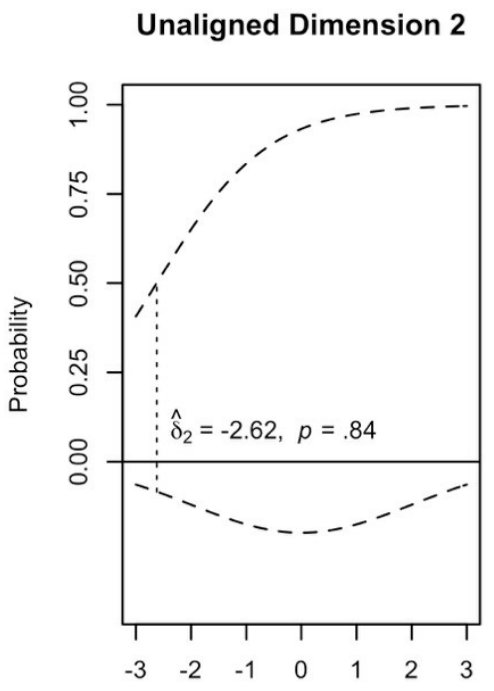

$\theta_{2}$
Aligned Dimensions 1 and 2

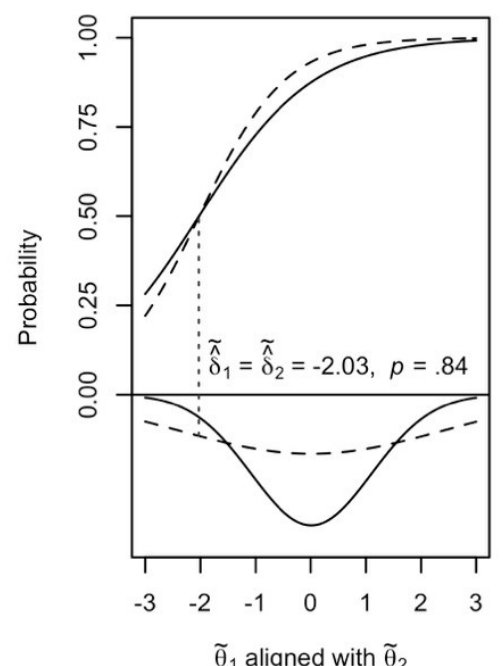

$\tilde{\theta}_{1}$ aligned with $\tilde{\theta}_{2}$

Figure 1. Illustration of scale alignment for two items on two dimensions with the same proportion-correct score $p$. After the scales have been brought into alignment, items with the same $p$ have the same difficulty estimate.

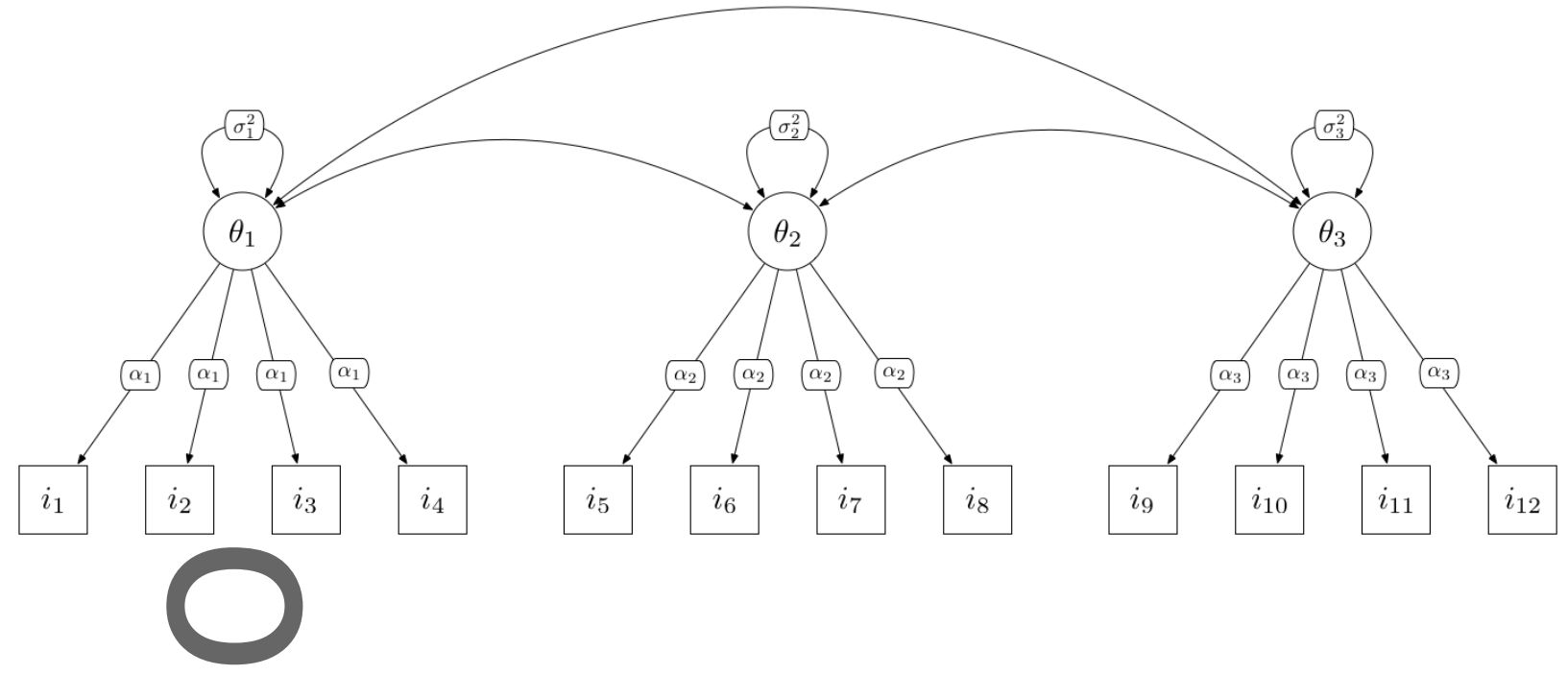

Figure 2. Path diagram of the between-items multidimensional Rasch model.

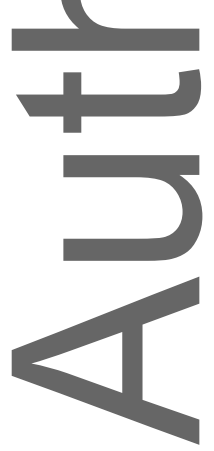

This article is protected by copyright. All rights reserved. 
Constrain Person Mean $=0$

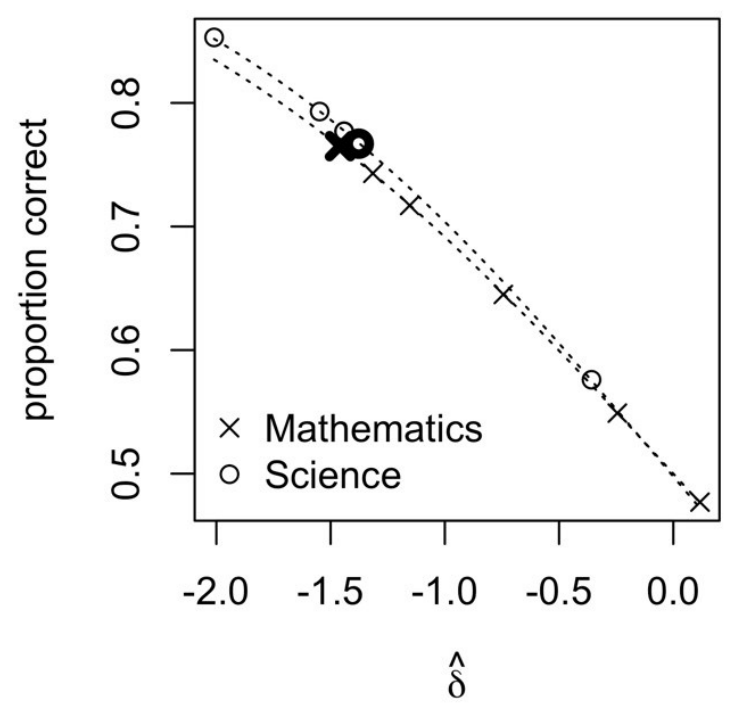

Constrain Item Mean $=0$

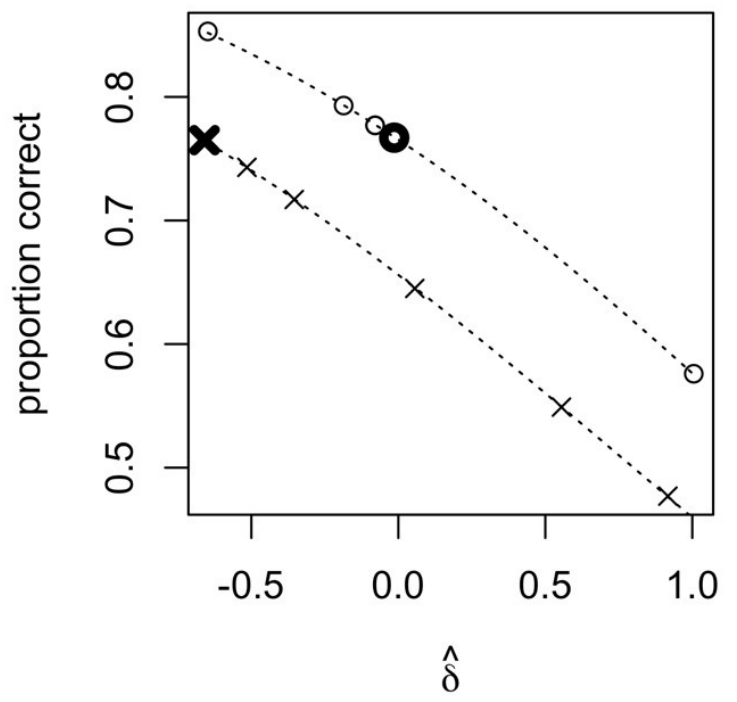

Figure 3. Illustration of the scale alignment problem. In each panel, estimated item difficulties are plotted against the observed proportion-correct scores. The highlighted mathematics item has a proportion-correct score of .765, and the highlighted science item has a proportion-correct score of .767 . In both panels, the estimated item difficulty is higher for the science item than for the mathematics item.

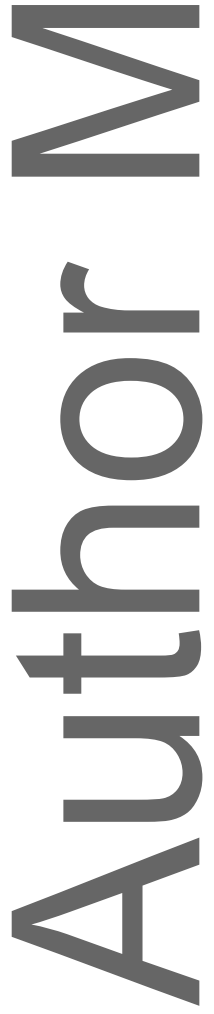

This article is protected by copyright. All rights reserved. 

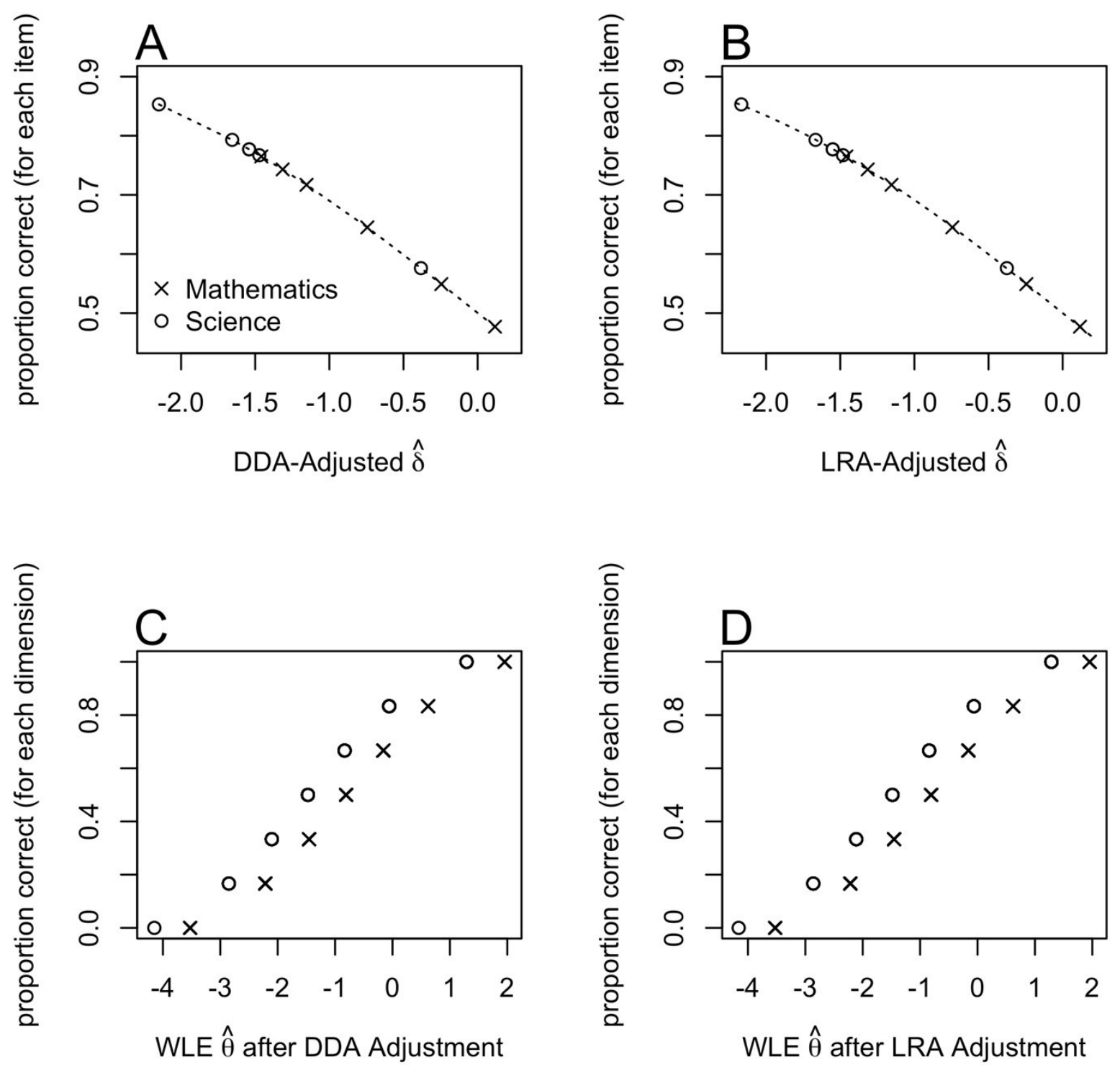

Figure 4. Scatter plots of sufficient statistics against item parameter estimates (Panels A and B) and person parameter estimates (Panels $\mathrm{C}$ and D) after DDA adjustment (Panels A and C) and LRA adjustment (Panels B and D).

This article is protected by copyright. All rights reserved. 


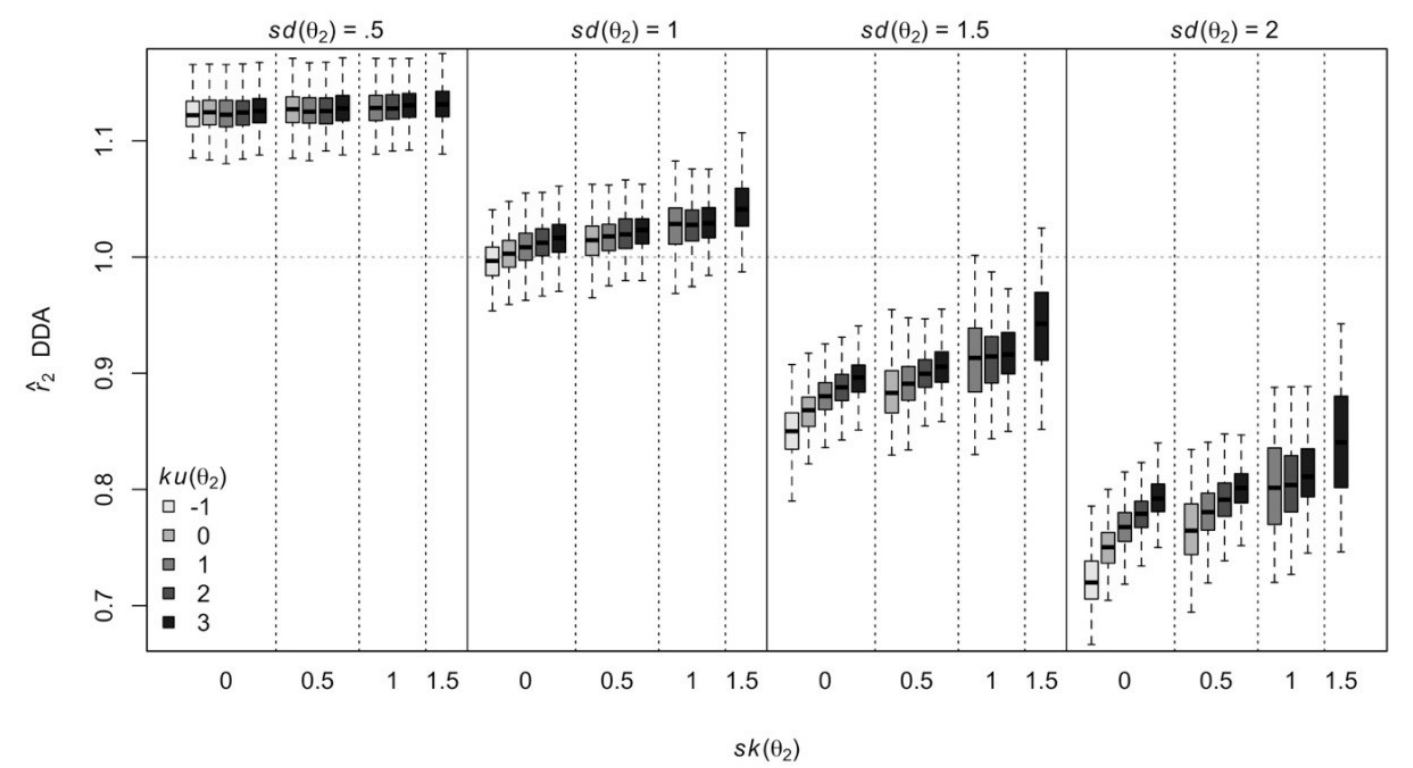

Figure 5. Box plots of the DDA $\hat{r}_{2}$ conditional on the standard deviation $(s d)$, skewness $(s k)$ and kurtosis $(k u)$ of the $\theta_{2}$ distribution.

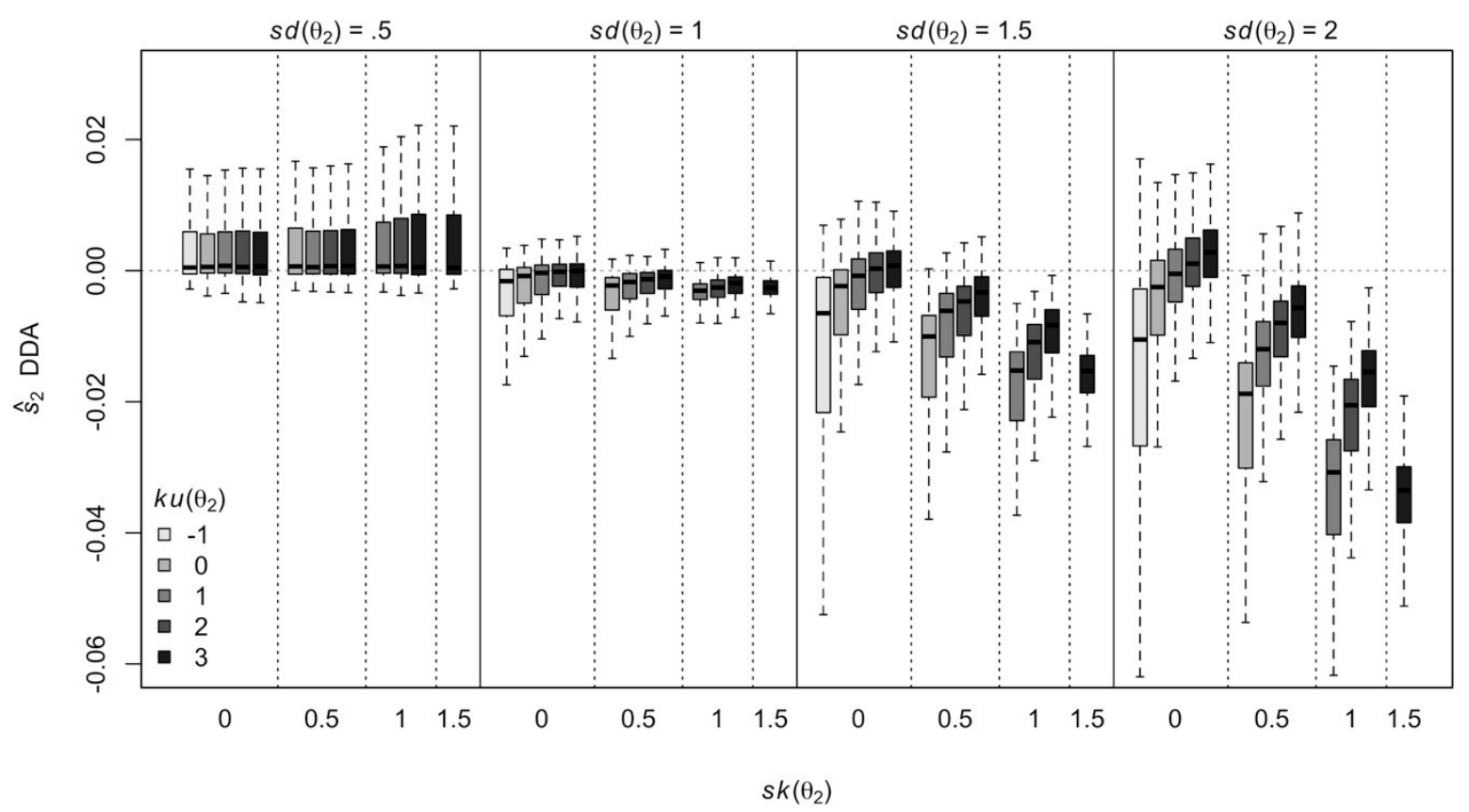

Figure Box plots of the DDA $\hat{s}_{2}$ conditional on the standard deviation (sd), skewness $(s k)$, and kurtosis $(k w)$ of the $\theta_{2}$ distribution.

This article is protected by copyright. All rights reserved. 


\section{University Library}

\section{- M M I N E R VA A gateway to Melbourne's research publications}

Minerva Access is the Institutional Repository of The University of Melbourne

Author/s:

Feuerstahler, L;Wilson, M

Title:

Scale Alignment in Between-Item Multidimensional Rasch Models

Date:

2019-06-01

Citation:

Feuerstahler, L. \& Wilson, M. (2019). Scale Alignment in Between-Item Multidimensional

Rasch Models. JOURNAL OF EDUCATIONAL MEASUREMENT, 56 (2), pp.280-301. https:// doi.org/10.1111/jedm. 12209 .

Persistent Link:

http://hdl.handle.net/11343/286875 